\title{
Monocular Image-based Time to Collision and Closest Point of Approach Estimation*
}

\author{
Peter Bauer ${ }^{1}$, Antal Hiba ${ }^{1}$, Balint Vanek ${ }^{1}$, Akos Zarandy ${ }^{1}$ and Jozsef Bokor ${ }^{1,3}$
}

\begin{abstract}
This paper deals with monocular image-based time-to-collision (TTC) and closest point of approach (CPA) estimation for aircraft sense and avoid. First, it proposes a disc-based pinhole camera projection model which can better represent a real 3D object. Then it proposes simple least squares optimal line fitting-based techniques for TTC and CPA estimation based-on measurable image parameters only. Possible errors in the image are considered through design nomograms and a collision decision threshold selection technique is presented. Theoretical results are verified through software-in-the-loop simulation and real flight test results. To the best of the author's knowledge the disc-based projection model and the line fit-based TTC and CPA estimation are new contributions in this field.
\end{abstract}

Index Terms-Sense and avoid, Monocular camera, Time to collision, Closest point of approach

\section{INTRODUCTION}

Sense and avoid (S\&A) capability is a crucial ability for the future unmanned aerial vehicles (UAVs). It is vital to integrate civilian and governmental UAVs into the common airspace according to [1] and [2]. At the highest level of integration (called Dynamic Operation in [2]) Airborne Sense and Avoid (ABSAA) systems are required to guarantee airspace safety.

In this field the most critical question is the case of noncooperative S\&A for which usually complicated multi-sensor systems are developed (see [3] for example). However, in case of small UAVs the size, weight and power consumption of the onboard S\&A system should be minimal. Monocular vision based solutions can be cost and weight effective therefore especially good for small UAVs [4], [5], [6], [7]. These systems basically measure the position (bearing) and size of intruder aircraft (A/C) camera image without range and intruder size information. This scale ambiguity makes the decision about the possibility of mid-air collision (MAC) or near mid-air collision (NMAC) complicated. Image-based time-to-collision (TTC) estimation methods are published in [8], [9], [10]. Here, TTC is defined as the time until the intruder crosses the plane of camera focal point irrespective of the side distance. So zero TTC does not trivially means a MAC. To decide about MAC or NMAC the side distance at

IEEE ID of final version: 978-1-4673-8345-5/16/\$31.00 (c)2016 IEEE, published in proceedings of IEEE MED'16 pp. 1168-1173

*This work is supported by the Office of Naval Research Global, Grant Number N62909-10-1-7081, Dr. Charles Holland program officer.

${ }^{1}$ Author is with Institute for Computer Science and Control, Hungarian Academy of Sciences (MTA SZTAKI), Budapest, Hungary Corresponding author: peter.bauerasztaki.mta.hu

${ }^{3}$ Author is with MTA-BME Control Engineering Research Group zero TTC should be somehow estimated. Because of the scale ambiguity its not possible to estimate the absolute distance however, the relative distance called closest point of approach (CPA) and defined in [5] and [11] can be estimated.

The current article targets to derive simple and reliable estimation methods for TTC and CPA considering the effect of 3D intruder objects onto camera projection rules and possible errors in camera image such as pixelization and threshold dependence of object detection. A NMAC / MAC (later called simply Collision) detection threshold selection methodology is also presented and results are demonstrated through Software-in-the-loop (SIL) simulation of several flight scenarios. It is assumed that both own aircraft and intruder fly along straight paths with constant velocity.

The article is divided into five sections. Section II summarizes the basic camera projection formulae, presents the ideas for simple TTC and CPA estimation and points out the problems if real 3D objects are projected to camera screen. Section III modifies the formulae to account for effects of $3 \mathrm{D}$ objects and reformulates TTC and CPA estimation accordingly. Section IV presents the proposed threshold selection method for Collision decision. Section V presents decision results based-on SIL scenario simulations. Finally section VIII concludes the paper.

\section{BASICS OF TTC AND CPA ESTIMATION}

The applied basic notations (image parameters) are shown in Fig. 1.

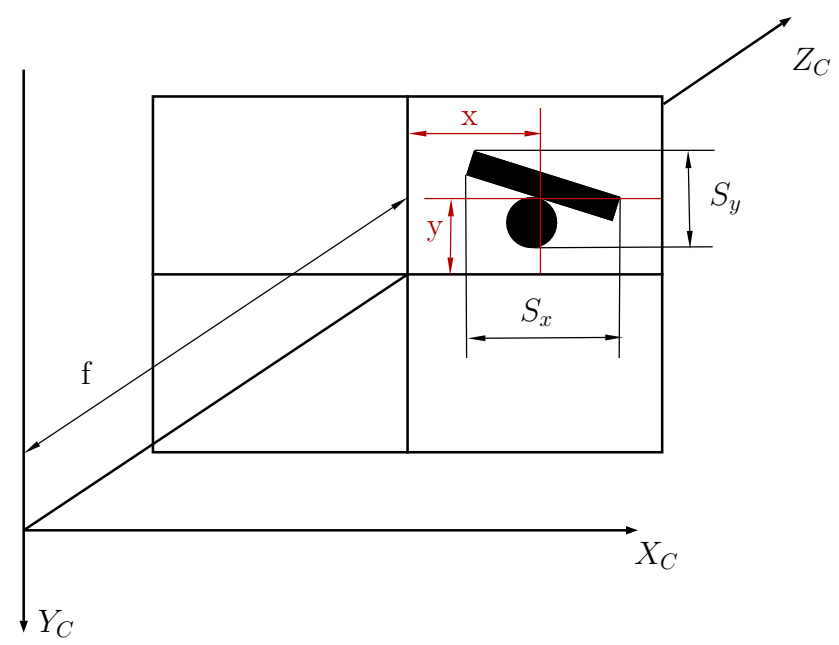

Fig. 1. Considered image parameters

In $X_{C}, Y_{C}, Z_{C}$ camera frame $x, y$ are the positions of 
intruder image centroid (IIC) and $S_{x}, S_{y}$ are the intruder image sizes (IIS) (horizontal / vertical). A pinhole camera model is used which relates image parameters $\left(x, y, S_{x}, S_{y}\right)$ to own aircraft camera focal length $f$, intruder position $\left(X, Y, Z\right.$ ) in camera frame, intruder size $R_{x / y}$ (horizontal / vertical), intruder relative velocities $V_{x}, V_{y}, V_{z}$ in camera frame, time to collision $t_{T C}$ (defined to go to zero as the aircrafts approach each other), miss distances at $\mathrm{Z}=0 X_{a}, Y_{a}$ and relative miss distances (CPA) $C P A=X_{a} / R_{x}$ or $Y_{a} / R_{y}$ The basic equations of pinhole camera projection model are:

Considering

$$
\begin{aligned}
& x=f \frac{X}{Z}, \quad y=f \frac{Y}{Z} \\
& S_{x}=f \frac{R_{x}}{Z}, \quad S_{y}=f \frac{R_{y}}{Z}
\end{aligned}
$$

$$
X=X_{a}-V_{x} t_{T C}, \quad Z=-V_{z} t_{T C}
$$

the above expressions can be reformulated. From now, formulae are presented only for the $x$ horizontal direction because the $y$ direction formulae are structurally the same that's why the $x$ indices are also neglected.

$$
x=-f\left(\frac{R}{V_{z}} \frac{C P A}{t_{T C}}-\frac{V_{x}}{V_{z}}\right), \quad S=-f \frac{R}{V_{z}} \frac{1}{t_{T C}}
$$

In [5] the ratio of $d x / d t$ and $d S / d t$ was used to estimate CPA. [11] pointed out that this ratio can be very uncertain in case of pixelization and other errors in $x$ and $S$ and their numerical differentiation. That's why it examined $d x / d t$ and $d S / d t$ separately and proposed thresholding of these values to decide about Collision. Large values of $d x / d t$ mean no threat of Collision meanwhile large values of $d S / d t$ mean that the intruder is very close to us. This led to a strategy which waits until $d x / d t$ violates the threshold and then decides about no threat of Collision. However, if $d S / d t$ violates the threshold earlier then an avoidance maneuver is done because intruder is close and there is a threat of Collision. However, this method can also magnify uncertainties in $x$ and $S$ because the calculation of time derivatives. So it would be better to decide about collision without applying time derivatives.

\section{A. Simple TTC and CPA estimation}

Taking a closer look at $S$ shows that its reciprocal is linearly proportional with $t_{T C}$ :

$$
\frac{1}{S}=-\frac{V_{z}}{f R} t_{T C}
$$

Here, $R, f$ and $V_{z}$ are constant in a given situation so (4) gives a simple linear relation between $1 / S$ and $t_{T C}$. However, on the right hand side both $\frac{V_{z}}{f R}$ and $t_{T C}$ are unknown. By substituting $t_{T C}=t_{C}-t$ where $t$ is actual time onboard the own aircraft and $t_{C}$ is the future time when $t_{T C}=0$ one gets a linear relation with known independent $(t)$ and dependent $\left(1 / S_{x}\right)$ variables:

$$
\frac{1}{S}=\underbrace{\frac{V_{z}}{f R}}_{a} t-\underbrace{\frac{V_{z}}{f R} t_{C}}_{b}
$$

Fitting a least squares optimal line to the registered $t(i)$ and $1 / S(i)(i=1: N)$ values its easy to estimate $t_{C}$ and so actual $t_{T C}(N)$ :

$$
t_{C}=-\frac{b}{a} \quad t_{T C}(N)=t_{C}-t(N)
$$

Examining now $x$ (see (3)) $S$ can be easily identified in it and this gives again a linear relation where one of the unknown parameters is CPA:

$$
x=S \cdot C P A+\underbrace{f \frac{V_{x}}{V_{z}}}_{c}
$$

So, the estimation of TTC and CPA only requires simple recursive LS optimal linear fits considering only the image centroid position $(x)$, size $(S)$ and time $t$. Similar method can be used in the vertical $(y)$ direction also. However, formulae in (1) are only valid for a line segment (length $R$ ) parallelly approaching the image plane. Resulting possible inaccuracies are discussed in the next subsection.

\section{B. Possible problems with $3 D$ objects}

Fig. 2 shows the possible problems of the projection models in (3) considering only a line parallelly approaching the image plane. With real $3 \mathrm{D}$ objects two problems can arise. One is the rotation of the object, the other is the depth information.

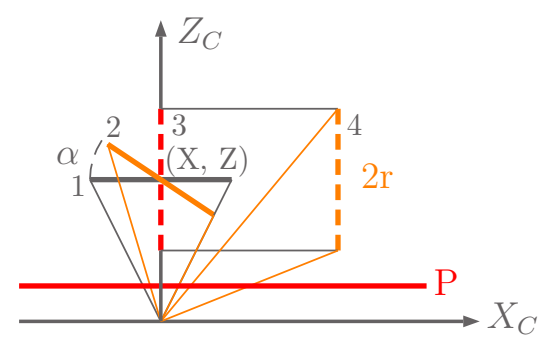

Fig. 2. Problems with parallel line formulae

In the figure $r$ denotes the half of the 'object' size ( $r=$ $R / 2), P$ is the image plane and $(\mathrm{X}, \mathrm{Z})$ is the position of the center point of the line in the $X_{C}, Z_{C}$ camera coordinate system. The same projection formulae as in (1) are derived considering the rotation of the object with angle $\alpha$ :

$$
\begin{aligned}
& x=f \frac{X \cdot Z+r^{2} \sin (\alpha) \cos (\alpha)}{Z^{2}-r^{2} \sin ^{2}(\alpha)} \\
& S_{x}=f \frac{2 Z \cdot r \cos (\alpha)+2 X \cdot r \sin (\alpha)}{Z^{2}-r^{2} \sin ^{2}(\alpha)}
\end{aligned}
$$

Substituting $\alpha=0$ and considering $2 r=R$ gives exactly (1). However, for nonzero $\alpha$ values the size and centroid position of the projected object will be different from (1) as (7) and 
the figure show (compare projected size of line 1 and 2). This means that rotation of a linear object (such as aircraft wing) will cause a change in its projection. $\alpha=90^{\circ}$ is again a special case where the line is parallel with the $\mathrm{Z}$ axis (see line 3). If the $X$ position of this line is zero, then its projected size is zero. However, if its X position is nonzero (line 4) then the projected size becomes nonzero. This means that the depth information gives a change in the size of the projected object. The effects of the change of the orientation and the depth information can be approximately described by a horizontal disc model instead of a simple line. Considering data about several aircraft from [12] the length/wingspan ratio gives a mean value of 0.93 which is not very far from 1 . This means that a disc can well approximate the horizontal contour of an aircraft. Detailed disc-based projection formulae and TTC / CPA estimation based-on these formulae are presented in the next section.

\section{DISC PROJECTION MODEL-BASED TTC AND CPA ESTIMATION}

This section summarizes the disc-based projection model and the TTC and CPA estimation method modified accordingly.

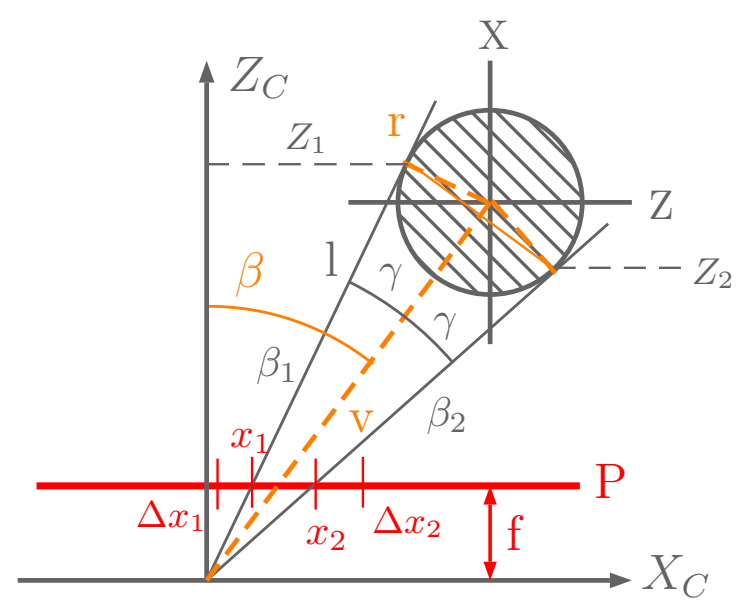

Fig. 3. Disc projection model

Fig. 3 shows the arrangement and notations used for the derivation of projection formulae $((\mathrm{X}, \mathrm{Z})$ disc center position, $P$ image plane, $r$ disc radius). The detailed derivation can be found in the appendix. The final properly approximated result is:

$$
\begin{aligned}
& S\left(\cos \left(\beta_{1}\right)+\cos \left(\beta_{2}\right)\right)=f \frac{2 R}{V_{z} t_{T C}} \\
& x\left(1-\frac{S^{2}\left(\cos \left(\beta_{1}\right)+\cos \left(\beta_{2}\right)\right)^{2}}{16 f^{2}}\right)= \\
& =S\left(\cos \left(\beta_{1}\right)+\cos \left(\beta_{2}\right)\right) \frac{C P A}{2}+f \frac{V_{x}}{V_{z}}
\end{aligned}
$$

Note that all $S, x, \beta_{1}$ and $\beta_{2}$ are features known from the image. So considering $\bar{S}=S\left(\cos \left(\beta_{1}\right)+\cos \left(\beta_{2}\right)\right)$ and $\bar{x}=x\left(1-\frac{S^{2}\left(\cos \left(\beta_{1}\right)+\cos \left(\beta_{2}\right)\right)^{2}}{16 f^{2}}\right)$ as corrected measured parameters leads to the same equations as (4) and (6). This means that the disc representation of the intruder object leads to measurable correction terms and does not affect the applicability of the TTC and CPA estimation method proposed in section II. The next section deals with possible errors and proposes a threshold selection methodology.

\section{POSSIBLE ERRORS AND THRESHOLD SELECTION}

The basic equations for TTC and CPA estimation from (8) are:

$$
\bar{S}=f \frac{2 R}{V_{z} t_{T C}}, \quad \bar{x}=\bar{S} \frac{C P A}{2}+f \frac{V_{x}}{V_{z}}
$$

As Fig. 3 and 7 shows there can be an error in the estimation of $x_{1}$ and $x_{2}$ points because of thresholding in camera object detection and pixelization. This error was experienced to be maximum 2 pixels in our system. We have modelled this error by a normally distributed random variable with variance $\sigma=0.7$ (this means a $3 \sigma$ bound of 2.1). The question is the effect of this error on the estimation of TTC and CPA.

Considering the image size, the error of $S$ is simply $\Delta x_{1}+\Delta x_{2}$ meanwhile the error of $\cos \left(\beta_{1}\right)+\cos \left(\beta_{2}\right)$ is more complicated. That's why it is considered that the error of $\bar{S}$ is also $\Delta x_{1}+\Delta x_{2}$. If equal absolute maximum errors are considered $\left(-\Delta x_{1}=\Delta x_{2}=\Delta x=3 \sigma>0\right)$ then the maximum error of $\bar{S}$ is $2 \Delta x$ and the minimum is $-2 \Delta x$. Considering $x$ its error is zero if the error of $S$ is symmetrical. Its largest error results if $\Delta x_{1}=\Delta x_{2}=\Delta x=$ $3 \sigma>0$. Considering $x=\left(x_{1}+\Delta x_{1}+x_{2}+\Delta x_{2}\right) / 2$ the largest $x$ error is $\Delta x$. However, $\bar{x}$ is different from $x$ and this should be considered by substituting the errors for $x$ and $\bar{S}$. After some manipulations considering the worst case values for every parameter the upper bound for $\bar{x}$ results as:

$$
\Delta \bar{x}=\frac{28}{16} \Delta x+\frac{12}{16 f}(\Delta x)^{2}+\frac{4}{16 f^{2}}(\Delta x)^{3}
$$

Finally, the lower (L) and upper (U) $3 \sigma$ bounds for the measured $\bar{S}$ and $\bar{x}$ curves can be derived as:

$$
\begin{aligned}
& \bar{S}_{L}=\frac{V_{z} t_{T C}}{2 f R+2 \Delta x V_{z} t_{T C}}, \quad \bar{S}_{U}=\frac{V_{z} t_{T C}}{2 f R-2 \Delta x V_{z} t_{T C}} \\
& \bar{x}_{L}=\bar{S} \frac{C P A}{2}-f \frac{V x}{V z}-2 \Delta x \frac{C P A}{2}-\Delta \bar{x} \\
& \bar{x}_{U}=\bar{S} \frac{C P A}{2}-f \frac{V x}{V z}+2 \Delta x \frac{C P A}{2}+\Delta \bar{x}
\end{aligned}
$$

The proposed method for threshold selection is to calculate these bounds and the nominal curves for a set of intruder aircrafts covering a wide range of size and velocity. Scenarios with fixed own aircraft velocity and camera parameters and with parallel A/C paths are considered. Additionally, 100 randomly disturbed curves can be generated from the nominal data applying the camera noise (with $\sigma$ variance) on $x_{1}$ and $x_{2}$ coordinates and deriving other parameters from them. TTC and CPA estimation through line fit is done for all curves considering a $t_{T C}$ range from about 10 to 1 second. TTC and CPA estimation errors are calculated in \% relative to the true values. 
From these calculations design nomograms can be plotted. One for the estimated TTC against real $t_{T C}$ and one for the CPA estimation error against real $t_{T C}$ again. The method of threshold selection is to first determine the estimated TTC threshold $\left(t_{T C}^{E}\right)$. Intersecting the curves of the TTC nomogram with this value gives the minimum and maximum real $t_{T C}$ values when the estimated one can be $t_{T C}^{E}$. By considering the resulted minimum and maximum $t_{T C}$ values the maximum CPA estimation error can be obtained from the other nomogram. After deciding about the minimum CPA below which avoidance should be done it should be increased by the maximum estimation error and that will be the CPA threshold.

In this work considered intruder aircraft sizes range from $1.2 \mathrm{~m}$ to $80 \mathrm{~m}$, and velocities range from $10 \mathrm{~m} / \mathrm{s}$ to $262 \mathrm{~m} / \mathrm{s}$ based-on the characterization of possible intruders published in [11]. Own A/C speed is selected to be $20 \mathrm{~m} / \mathrm{s}$ (small UAV) and camera focal length to be $f=850$. Nomograms were plotted from the bounds (bound-based $=\mathrm{BB}$ selection) and from the minimum / maximum (real-based $=\mathrm{RB}$ selection) and mean (mean real-based MRB selection) differences of the 100 random patterns. They showed that an $1.2 \mathrm{~m}$ intruder can not be handled with such camera focal length (first detection time is too close to $t_{T C}^{E}$ for the estimates to converge) that's why results for $3.5 \mathrm{~m}$ intruder and above are plotted only.

In our case $t_{T C}^{E}=2 \mathrm{sec}$ was selected as decision time and $C P A=10$ was decided as a limit for avoidance. Note this means that every intruder is avoided which is closer to us then its wingspan times 10 . This makes the activation of avoidance self scaling.

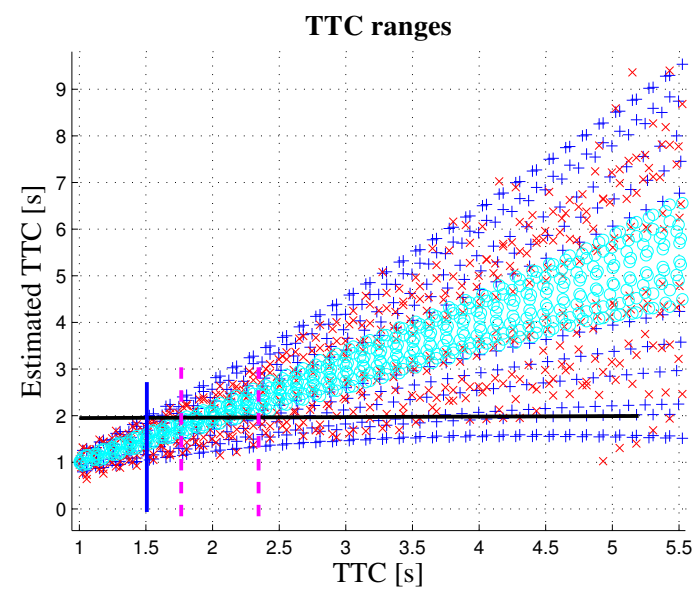

Fig. 4. Nomogram for TTC limit selection (blue +: bound-based, red cross: real-based, cyan circle: mean real-based)

Figs. 4 and 5 show the selection of thresholds. The horizontal line in Fig. 4 is the $2 \mathrm{sec}$ limit for the estimated TTC, the vertical lines are the projection lines from the intersection with different nomograms to the real $t_{T C}$ (continuous line from the bound-based, dashed lines from the mean real-based nomograms). In Fig. 5 the dashed lines are the projection lines from the $t_{T C}$ values selected in Fig. 4 to the CPA error nomogram. Their intersection with the upper curve of cyan circles should be considered as the maximum CPA error at that time. The results are summarized in Table I. $\infty$ means that there is no intersection of $t_{T C}^{E}$ with the curve of lowest estimated TTC values (see Fig. 4). Note that $M I N\left(t_{T C}\right.$ is the worst case time to collision when the decision about avoidance will be done. This should be compared to the meanuvering capabilities of the own $\mathrm{A} / \mathrm{C}$ and if avoidance is impossible during this time, $t_{T C}^{E}$ should be increased. $C P A_{L I M}$ is the finally selected limit CPA value from the given nomogram. This shows that the bounds are the most conservative. Considering randomly generated data gives lower limits for the CPA error and of course results from the mean random data are the most optimistic. In the next section all three selected bounds will be extensively tested in SIL simulation scenarios.

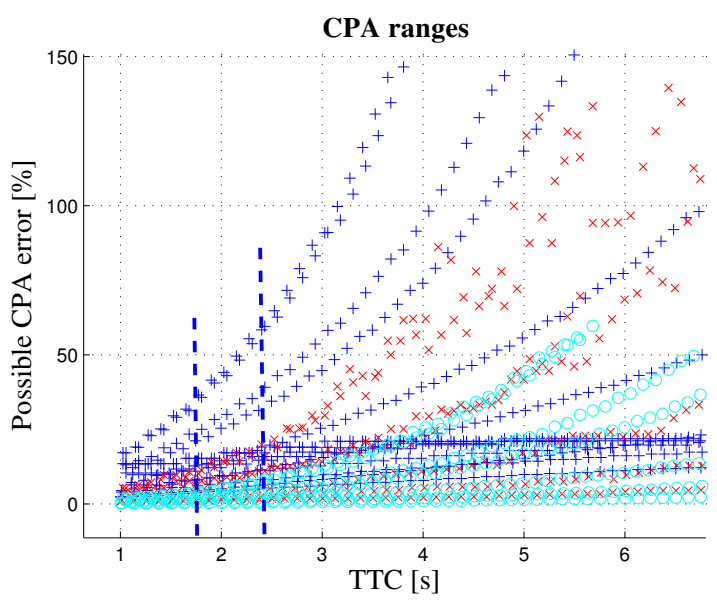

Fig. 5. Nomogram for CPA limit selection (blue +: bound-based, red cross: real-based, cyan circle: mean real-based)

TABLE I

THRESHOLD SELECTION RESULTS

\begin{tabular}{|c||c|c|c|c|}
\hline Nomogram & & TTC & CPA error & $C P A_{L I M}$ \\
\hline $\begin{array}{c}\text { Bound- } \\
\text { based }\end{array}$ & $M I N\left(t_{T C}\right)$ & 1.525 & $30 \%$ & \\
\hline Real- & $M A X\left(t_{T C}\right)$ & $\infty$ & $360 \%$ (for $4.8 \mathrm{~s})$ & 36 \\
based & $M I N\left(t_{T C}\right)$ & 1.6 & $11 \%$ & 19 \\
\hline Mean real- & $M I N\left(t_{T C}\right)$ & $\infty$ & $90 \%$ (for $4 \mathrm{~s})$ & 19 \\
based & $M A X\left(t_{T C}\right)$ & 1.8 & $4 \%$ & 11 \\
\hline
\end{tabular}

\section{SIL SIMULATION TEST CAMPAIGN}

The same SIL simulation environment is applied as in [11] by having ascending / descending straight intruder paths from left and right of own aircraft. The camera $f p s$ is set to 8 and random noises are generated on the 'measured' $S$ and $x$ values. No avoidance maneuver was executed, only the decisions were tested. The simulation campaign is run for five different intruder aircraft sizes (wingspan) $(3.5 \mathrm{~m}$, $10 \mathrm{~m}, 20 \mathrm{~m}, 40 \mathrm{~m}$ and $60 \mathrm{~m}$ ) ranging from small UAV through general aviation Cessna to large transport / airliner. Three different velocity cases (minimum, mean and maximum) are run for each $\mathrm{A} / \mathrm{C}$ based-on the characterization of possible 
intruders published in [11]. In every simulation case (given intruder size and velocity) 35 different scenarios (intruder directions) are tested. The test CPA values are $0,10,20$ and 40. The goal of the design was to have no missed detection (MD) for $\mathrm{CPA}=10$ and below. If the estimated TTC is below the $2 \mathrm{sec}$ threshold collision decision is done based-on the BB, RB and MRB CPA thresholds also. Results are summarized in Table II by calculating the percentage of MDs and false alarms (FAs) for the overall 525 simulated scenarios.

TABLE II

SIL SIMULATION RESULTS

\begin{tabular}{|c||c|c||c|c||c|c||c|c|}
\hline Nom. & CPA & 0 & CPA & 10 & CPA & 20 & CPA & 40 \\
\hline & MD & FA & MD & FA & MD & FA & MD & FA \\
\hline BB & 0 & 0 & 0 & 0 & 0 & 100 & 0 & 7.4 \\
\hline RB & 0 & 0 & 0 & 0 & 0 & 15.6 & 0 & 0 \\
\hline MRB & 0 & 0 & 45 & 0 & 0 & 0.8 & 0 & 0 \\
\hline
\end{tabular}

The table shows that the real random curves-based threshold selection is the best because, the mean real-based has $45 \% \mathrm{MD}$ for $\mathrm{CPA}=10$ which is unacceptable, and the boundbased has $7.4 \% \mathrm{FA}$ also for $\mathrm{CPA}=40$. The $\mathrm{RB}$ threshold gives a $15.6 \%$ FA for $\mathrm{CPA}=20$ which can be acceptable and is not surprising considering the $C P A_{L I M}=19$ threshold which is very close to 20 .

Another issue is the real $t_{T C}$ when the decisions are done. This ranges from 0.8 seconds to 5-6 seconds which shows that late and early decisions are also possible. For $\mathrm{CPA}=0$ the minimum value is 1.5 seconds which is about the selected minimum value from the nomogram. The possibly problematic cases are the $0.8 \mathrm{sec}$ for $\mathrm{CPA}=10$ and above but in these cases the intruder is farther from own $\mathrm{A} / \mathrm{C}$ and so, the avoidance can be also possible.

The next section briefly introduces the vision system and methods applied onboard our UAV in S\& A flight test experiences (for details of the UAV see [13]).

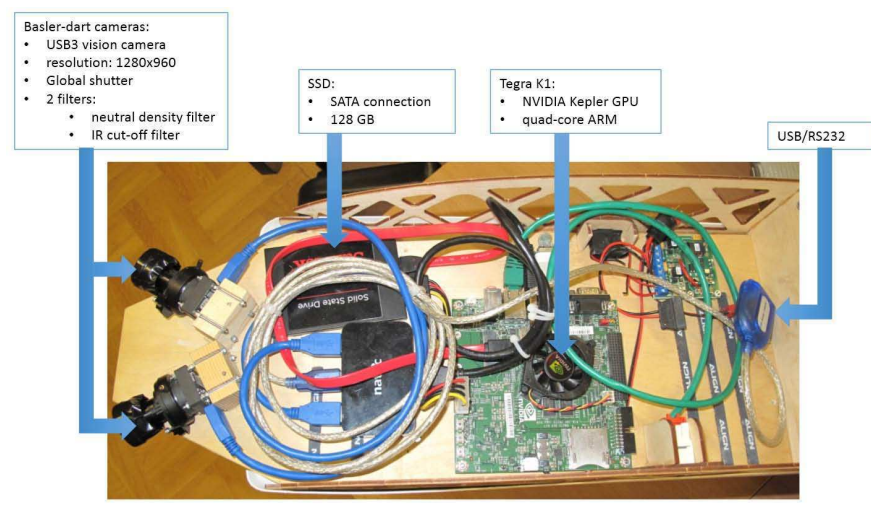

Fig. 6. Camera system mounted on Sindy aircraft.

\section{CAMERA SYSTEM}

Real-time object detection, classification and tracking are essencial in an SAA system. Our experimental setup for image processing is based on the nVidia Jetson TK1 development board which consists of the TK1 SoC with the necessary peripherals (SATA, GigE, HDMI, USB, GPIO) and can handle two HD cameras (Fig. 6). This is a low power system with a quad-core ("4-Plus-1") ARM Cortex A15 and a Kepler GPU with 192 CUDA cores. The power consumption is $5-10 \mathrm{~W}$ which is suitable for a small UAV.

The object detection algorithm is the improved version of the small dense object detector presented in [14]. After a trigger signal, the aircraft control provides the Euler angles (Yaw, Pitch, Roll) of the UAV body system and the two HD cameras aquire the visual information. The GPU starts to compute the necessary convolution and morpholigic operations on the two HD images, while the quad-core ARM computes large object masks on subsampled small sized images. Horizon estimation and threshold updates are also computed by the ARM part of the processor. The horizon estimates are corrected based on the images, which makes it possible to create a better ground mask. The current visual system can detect UAVs only on the sky.
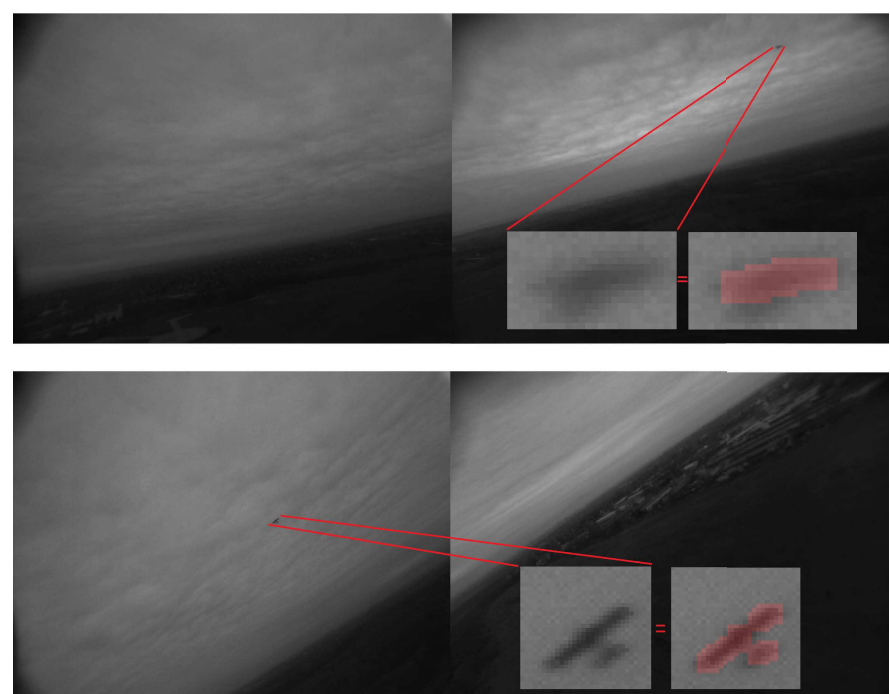

Fig. 7. Object detection exapmples. Optical transmission in real environment has large disturbance (air, non-ideal optics) which increases object size estimation error above 1 pixel even with a good object detector.

The result of the preprocessing phase is a binary image which contains only sky objects. Sky objects are not always aircrafts. A classification is required which eliminates false objects for instance cloud edges. After classification, the remaining objects are tracked and their projected trajectories are analysed. In Fig (??) the trajectory of a small UAV is pesented with its projected trajectory. The covering rectangle of the tracked object is projected to a virtual camera which depth axis is identical to the desired moving direction of the UAV. Projection is necessary because the cameras are placed on the aircraft in different orientations. Furthermore, the real orientation of the UAV body can be different from the desired direction because of wind or periodical path control errors, while the UAV moves to its desired direction in general. In the later, we use this unified virtual camera for size and position measurement.

When we examine the error of TTC and CPA esimates we need the deviation of measured position and size values 
assuming the mean is the accurate value. In Fig. (7) two objet detection examples ae shown where the scale of detection errors can be seen. Theoretically only the pixelization error disturbes the size and position calculation, however, the air and non-ideal optics increase the detection error in real situations. Even a small mist can cause heavy blur effect on the captured image which makes the accurate size estimation impossible. Cloud shadows and other artifacts can cause further size and position errors in detection, which affect the TTC and CPA estimations.

The next section presents the first application of the developed TTC and CPA estimation method in real flight tests.

\section{REAL FLIGHT TEST RESULTS}

Flight tests with the above described camera system and with an $1.2 \mathrm{~m}$ wingspan intruder were conducted prescribing parallel straight paths in $20 \mathrm{~m}$ and $50 \mathrm{~m}$ distance. This means test of the method with $C P A \approx 17$ and $C P A \approx 42$. The $1.2 \mathrm{~m}$ intruder wingspan means a critical case as was pointed out in Section IV. Another problem is the loose tracking of paths by the aircrafts which violates the assumption of straight flight paths. Despite these critical circumstances the results are promising as shown in Fig. 8. The estimated CPA values of close and far intruders are clearly distinguishable in the range of 2 to $0 \mathrm{sec}$. estimated TTC. What is more the estimated CPA values are close to the prescribed ones (15-20 for $\mathrm{CPA}=17$ and 40-50 for $\mathrm{CPA}=42$ ).

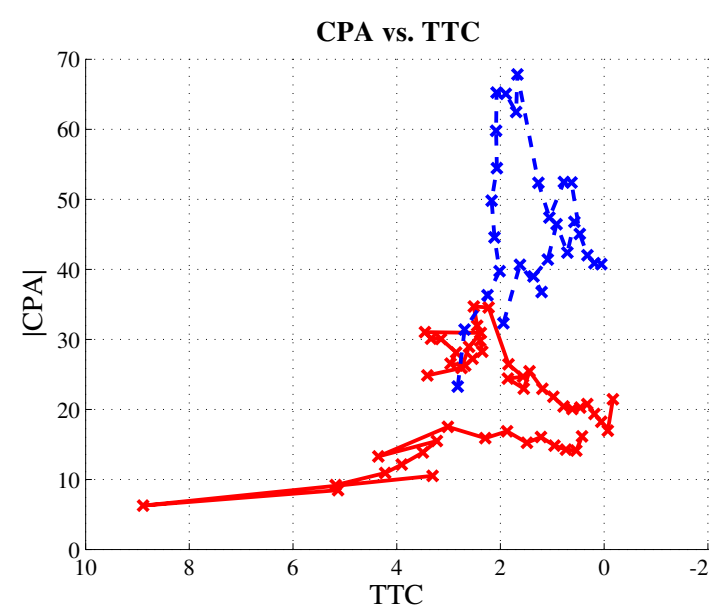

Fig. 8. TTC-CPA diagram from real flight test estimates (red continuous line for $\mathrm{CPA}=17$ scenarios, blue dashed line for $\mathrm{CPA}=42$ ones)

\section{CONCLUSION}

APPENDIX

\section{DERIVATION OF DISC-BASED PROJECTION FORMULAE}

During the image processing, the contour of the intruder image is identified and size (in $\mathrm{X}$ and $\mathrm{Y}$ directions) is calculated based-on minimum / maximum contour coordinates in each direction. The position is calculated as the centroid of the contour. Considering the disc model in the $\mathrm{X}-\mathrm{Z}$ plane of camera frame the projected contour points are $x_{1}$ and $x_{2}$ and so $S=x_{2}-x_{1}$ and $x=\frac{x_{2}+x_{1}}{2}$. So, the first task is to derive expressions for $x_{1}$ and $x_{2}$. Based on Fig. 3 they can be expressed as:

$$
\begin{aligned}
& x_{1}=f \tan \left(\beta_{1}\right), \quad x_{2}=f \tan \left(\beta_{2}\right) \\
& \beta_{1}=\beta-\gamma, \quad \beta_{2}=\beta+\gamma
\end{aligned}
$$

Considering that the lines intersecting the $P$ plane at $x_{1}$ and $x_{2}$ are the tangents of the disc, the tangents of the angles can be formulated as shown:

$$
\begin{aligned}
& v=\sqrt{X^{2}+Z^{2}}, \quad l=\sqrt{X^{2}+Z^{2}-r^{2}} \\
& \tan (\beta)=\frac{X}{Z}, \quad \tan (\gamma)=\frac{r}{l} \\
& \tan \left(\beta_{1}\right)=\tan (\beta-\gamma), \quad \tan \left(\beta_{2}\right)=\tan (\beta+\gamma) \\
& \tan \left(\beta_{1}\right)=\frac{\tan (\beta)-\tan (\gamma)}{1+\tan (\beta) \tan (\gamma)}=\frac{X l-r Z}{Z l+X r} \\
& \tan \left(\beta_{2}\right)=\frac{\tan (\beta)+\tan (\gamma)}{1-\tan (\beta) \tan (\gamma)}=\frac{X l+r Z}{Z l-X r}
\end{aligned}
$$

Combining (11) and (12) $S$ and $x$ finally result as:

$$
S=f \frac{2 r l}{Z^{2}-r^{2}}, \quad x=f \frac{X Z}{Z^{2}-r^{2}}
$$

Substituting $l$ from (12) and the $X, Z$ distances from (2) results in overly complicated expressions from which $t_{T C}$ (or $t_{C}$ ) and CPA can not be easily estimated. However, making a simplification which is negligible in practical applications makes the formulae similarly simple as they were.

Considering the $Z_{1}$ and $Z_{2}$ coordinates in Fig. 3 they can be constructed as $Z_{1}=Z-\Delta Z+\Delta r$ and $Z_{2}=Z-\Delta Z-$ $\Delta r$. This leads to the expression:

$$
\cos \left(\beta_{1}\right)+\cos \left(\beta_{2}\right)=\frac{2 Z-2 \Delta Z}{l}
$$

Considering other relations in Fig. $3 \Delta Z$ results as:

$$
\Delta Z=\frac{r^{2} Z}{X^{2}+Z^{2}}
$$

$\Delta Z$ is the projection of the line segment between point $(\mathrm{X}, \mathrm{Z})$ and the intersection point of $\overline{Z_{1} Z_{2}}$ with $v$ to the $\mathrm{Z}$ axis. Substituting this into (14) finally $l$ can be expressed with $X, Z, r, \beta_{1}, \beta_{2}$. However, substituting this expression into (13) gives again overly complicated expressions. The solution is the approximation of $\Delta Z$ as follows:

$$
\Delta Z^{\prime}=\frac{r^{2}}{Z}, \quad\left(\Delta Z^{\prime} \geq \Delta Z\right)
$$

This means that the effect of $X^{2}$ is neglected. An intruder should be close in the $X$ direction to be a real threat of MAC / NMAC so this neglection can be reasonable. However, examine the magnitude of neglection closer.

$$
\Delta Z^{\prime}-\Delta Z=\frac{r^{2} X^{2}}{Z\left(X^{2}+Z^{2}\right)}
$$

Here, $r^{2}$ is constant in a given scenario, the others are timevarying. If $\frac{X^{2}}{Z\left(X^{2}+Z^{2}\right)}$ is close to 0 that means that the error 
is negligible. At first glance, its hard to state that it is close to zero. However, consider its difference from 1 :

$$
1-\frac{X^{2}}{Z\left(X^{2}+Z^{2}\right)}=\frac{(Z-1) X^{2}+Z^{3}}{Z X^{2}+Z^{3}}
$$

If $Z \gg 1$ then this difference is about 1 which means that $\frac{X^{2}}{Z\left(X^{2}+Z^{2}\right)}$ is about zero. $Z=1$ means that the intruder is $1 \mathrm{~m}$ in front of own aircraft and its too late to make any decision. So, in the time slot when the Collision decision should be made $Z \gg 1$ is surely satisfied. This means that the error of the approximation of $l$ is negligible in the practical range of parameters.

Substituting (14) and (15) into (13), considering $R=2 r$ and reordering terms in $x$ gives the final approximated formulae for $S$ and $x$ in (8).

\section{REFERENCES}

[1] "Roadmap for the integration of civil Remotely-Piloted Aircraft Systems into the European Aviation System," European RPAS Steering Group, Tech. Rep., 2013.

[2] Department of Defense, USA, "Unmanned Aircraft System Airspace Integration Plan," March 2011.

[3] L. R. Salazar, R. Sabatini, S. Ramasamy, and A. Gardi, "A Novel System for Non-Cooperative UAV Sense-And-Avoid," in In Proceedings of European Navigation Conference 2013 (ENC 2013), April 2013.

[4] B. Vanek, T. Peni, A. Zarandy, J. Bokor, T. Zsedrovits, and T. Roska, "Performance Characteristics of a Complete Vision Only Sense and Avoid System," in in Proceedings of AIAA GNC 2012 (Guidance, Navigation and Control Conference), no. AIAA 2012-4703, Minneapolis, Minnesota, August 2012, pp. 1-15.

[5] S. D. B, "Reactive Image-based Collision Avoidance System for Unmanned Aircraft Systems," Master's thesis, Australian Research Centre for Aerospace Automation, May 2011.

[6] Y. Watanabe, "Stochastically Optimized Monocular Vision-based Navigation and Guidance," Ph.D. dissertation, Georgia Institute of Technology, 2008.

[7] E. W. Frew, "Observer Trajectory Generation for Target-Motion Estimation Using Monocular Vision," Ph.D. dissertation, Stanford University, 2003.

[8] F. Meyer and P. Bouthemy, "Estimation of time-to-collision maps from first order motion models and normal flows," in In Proc. of 11th IAPR International Conference on Pattern Recognition, 1992.

[9] J. Byrne and C. J. Taylor, "Expansion Segmentation for Visual Collision Detection and Estimation," in In Proc. of IEEE International Conference on Robotics and Automation, 2009.

[10] A. Schaub and D. Burschka, "Spatio-Temporal Prediction of Collision Candidates for Static and Dynamic Objects in Monocular Image Sequences," in In Proc. of IEEE Intelligent Vehicles Symposium (IV 2013), 2013.

[11] P. Bauer, B. Vanek, T. Peni, A. Futaki, B. Pencz, A. Zarandy, and J. Bokor, "Monocular image parameter-based aircraft sense and avoid," in In proceedings of 23rd Mediterranean Conference on Control and Automation (MED'15), Torremolinos, Spain, 2015.

[12] [Online]. Available: http://www.airliners.net/aircraft-data/

[13] B. Vanek, P. Bauer, I. Gozse, M. Lukatsi, I. Reti, and J. Bokor, "Safety critical platform for mini UAS insertion into the common airspace," in In Proc. of AIAA Guidance, Navigation and Control Conference 2014, 2014.

[14] Á. Zarándy, M. Nemeth, Z. Nagy, A. Kiss, L. Santha, and T. Zsedrovits, "A real-time multi-camera vision system for uav collision warning and navigation," Journal of Real-Time Image Processing, pp. $1-16,2014$. 